\title{
Protein-Based Textiles: Bio-Inspired and Bio- Derived Materials for Medical and Non-Medical Applications
}

\section{Citation}

Deravi, Leila F., Holly M. Golecki, and Kevin Kit Parker. 2013. “Protein-Based Textiles: BioInspired and Bio-Derived Materials for Medical and Non-Medical Applications." Journal of Chemical and Biological Interfaces 1 (1) (April 1): 25-34. doi:10.1166/jcbi.2013.1009.

\section{Published Version}

doi:10.1166/jcbi.2013.1009

\section{Permanent link}

http://nrs.harvard.edu/urn-3:HUL.InstRepos:17985227

\section{Terms of Use}

This article was downloaded from Harvard University's DASH repository, and is made available under the terms and conditions applicable to Open Access Policy Articles, as set forth at http:// nrs.harvard.edu/urn-3:HUL.InstRepos:dash.current.terms-of-use\#OAP

\section{Share Your Story}

The Harvard community has made this article openly available.

Please share how this access benefits you. Submit a story.

\section{Accessibility}




\title{
Protein-based Textiles: Bio-inspired and Bio-derived Materials for Medical and Non- Medical Applications
}

Leila F. Deravi, Holly M. Golecki, and Kevin Kit Parker

Disease Biophysics Group, Wyss Institute for Biologically Inspired Engineering, School of Engineering and Applied Sciences, Harvard University, Cambridge, MA 02138

\section{Corresponding author:}

\author{
Kevin Kit Parker \\ Harvard School of Engineering and Applied Sciences \\ 29 Oxford St (Rm 321) \\ Cambridge, MA 02138 \\ Phone: (617) 495-2850 \\ Fax: (617) 496-1793 \\ E-mail: kkparker@seas.harvard.edu
}




\begin{abstract}
The hierarchical structure-dependent function of self-assembling proteins regulates the biochemical and mechanical functions of cells, tissues, and organs. These multi-scale properties make proteins desirable candidates for novel supramolecular materials that require tailored properties and customizable functions. The ability to translate molecular domains of proteins into the bulk production of conformable materials, such as textiles, is restricted by the current limitations in fabrication technologies and the finite abundance of protein starting material. We will review the common features of self-assembling proteins, including their structure-dependent mechanical properties and how these characteristics have inspired techniques for manufacturing protein-based textiles. These technologies coupled with recent advances in recombinant protein synthesis enable the bulk production of fibers and fabrics that emulate the hierarchical function of natural protein networks.
\end{abstract}

\title{
Introduction
}

In mammals, protein networks assemble to regulate the form and function of cells and tissues while maintaining the structural stability of coupled organ systems. ${ }^{1-3}$ Cells secrete and assemble proteins and, either alone or in coordination with other cells, build nanoscale fibrous structures and networks with chemical and mechanical anisotropy. ${ }^{4,5}$ Many other organisms manufacture and utilize protein networks as ex vivo tools, for defense, transportation, to capture prey, and to protect offspring. ${ }^{6-8}$ Understanding the underlying design principles of how proteins assemble themselves into hierarchical fibrous structures can provide researchers with new insights into designing, building, and testing protein-based textiles. These synthetic systems can be a desirable tool for a variety of applications in medicine and industry. 
Protein textiles are envisioned as a system of multiple mechanosensitive protein domains, which are strung together into macromolecular assemblies of fibers by a fabrication process that does not compromise their mechanical properties. Biomanufacturing of protein networks is accomplished by cells or specialized organs in animals and insects., 5, 7, 9-12 For example, silkworms or spiders produce silk fibers for cocoons or webs through a step-wise process mediated by an interplay between shear forces, $\mathrm{pH}$, and ionic strength beginning in their major ampullate gland. ${ }^{6,8,12,13}$ Silk fibers can then be manually isolated from cocoons, where raw silk fibers are then reeled, twisted, or doubled to make a thread. ${ }^{7,}{ }^{14}$ In addition to their ability to withstand robust manufacturing conditions, protein textiles offer several advantages over conventional polymer textiles composed of nylon, polyesters, or vinylon, such as high extensibilities without failure, biocompatibility, and tunable stiffness. ${ }^{7,9,15,16}$ The structuredependent functional properties of protein-based textiles make them a desirable material for a variety of applications that require programmable chemical and mechanical features, such as elasticity, hydrophobicity, and conformability, for clothing, wound dressings, surgical sutures, body armor, and filters.

Recently, recombinant protein technologies have been optimized to manufacture proteins independent of the natural organism. ${ }^{17-19}$ Synthesized proteins can be processed using traditional manufacturing techniques, such as wet spinning or electrospinning, to produce protein based fibers for medical applications, such as vascular grafts or tissue engineered scaffolds. ${ }^{20-22}$ In this review, we will examine the design rules mediating protein self-assembly on the molecular level, and the current technologies available to build protein-based textiles, and the tools available to test these properties across multiple spatial scales. 


\section{Designing Protein Textiles}

The spatial scales of a protein textile, spanning from approximately the nanometer to centimeter length scales, is depicted in Figure 1. A feature of proteins is their hierarchical arrangement, beginning with the coordinated assembly of single amino acids to form peptides of the primary protein structure. ${ }^{2,23}$ This primary structure contains some combination of the twenty amino acids and their post-translational counterparts that are assembled through non-covalent hydrogen bonding. ${ }^{7}$ This ensemble of peptides is further stabilized by structural folding that yields a defined secondary structure, which is also held together by non-covalent hydrogen bonds. There are three common secondary structure motifs that impact protein stability: $\alpha$-helix, $\beta$-sheet, and random coil (Figure 1). ${ }^{7,23}$ Both $\alpha$-helices and $\beta$-sheets are stabilized by hydrogen bonding or hydrophobic core interactions that lock their structures into place. Random coils, on the other hand, are not stabilized by hydrogen bonding and exhibit an irregular secondary structure, which decreases the stability and overall stiffness of random coil proteins. ${ }^{7}$ The secondary structure also influences final protein conformation (tertiary structure) and their arrangement (quaternary structure) in the microenvironment of biological tissues. ${ }^{7,}{ }^{24}$ Protein fibers are polymerized through non-covalent bonding at the protein's N-termini, where supramolecular networks aggregate via electrostatic bonds to form the extracellular matrix (Figure 1). ${ }^{5,25}$ This system of secondary, tertiary, and quaternary folding across multiple spatial scales ensures that mechanical proteins remain stable and their assembly into a centimeter-scale network of fibers does not lose its mechanical integrity in extracellular space.

Collagen, Fibronectin $(\mathrm{FN})$, and silk are three proteins that assemble under different conditions but possess a mechanically stable secondary structure that contributes to their biological function. Collagen, which is the most abundant extracellular matrix (ECM) protein in 
animals, functions to maintain strength and elasticity of tissues, blood vessels, ligaments, and bone. $^{25,26}$ It is a linear polypeptide containing a repetitive primary structure comprised of $(\mathrm{GXY})_{\mathrm{n}}$ repeats, where $X$ and $Y$ can be any amino acid including Glycine $(\mathrm{G})$, Proline (P), or hydroxyproline. ${ }^{25}$ This repetitive structure influences intramolecular folding and the assembly of three coiled $\alpha$-helices twisted to form a super helix quaternary structure. ${ }^{16}$ In its super helix conformation, collagen assembles into fibrils through non-covalent bonding at their N-terminus, resulting in a fibrillar elastic modulus ranging from 0.6 (hydrated) to 3.2 (dry) GPa with maximum strain before failure of $\sim 30 \% .^{26,27}$ These fibrils assemble into supramolecular complexes, where the final diameter is regulated by the function of the specific tissue or organ. ${ }^{25}$, ${ }^{26}$ For example, collagen fibrils with diameters of $20 \mathrm{~nm}$ are arranged orthogonally in the cornea to maintain its structure while retaining optical transparency. Larger-diameter (500nm) fibrils align in parallel bundles to support the high tensile demands of mature tendons. ${ }^{25-27}$ Thus, the repetitive primary structure of collagen, its coordinated self-assembly, and the demands from its local microenvironment give rise to its ultimate biological function.

Another ECM protein, FN is structurally and functionally different from collagen, but it utilizes similar design principles to form fibrils in vivo. $\mathrm{FN}$ is a multidomain glycoprotein (450 $\mathrm{kDa}$, whose primary structure contains three (I, II, or III) repeating $\beta$-sheet structures. ${ }^{4,5,10} \mathrm{FN} \mathrm{I}$ and II domains contain interchain disulfide bonds that do not unfold. The FN III domain does not contain disulfide bonds, which enables FN to be manipulated by cells without breaking. ${ }^{28}$ FN exists in two quaternary conformations: a native, globular conformation, which occurs in the absence of external tension, and an extended conformation that is unfolded FN. ${ }^{4,28,29}$ In this extended conformation, FN recruits other globular molecules from extracellular space, initiating polymerization via non-covalent bonding at its N-terminus. ${ }^{4,29}$ Fibrillar FN is not static once it is 
coupled to cells; it can be rearranged, remodeled, and recycled to meet the demands of its local microenvironment. ${ }^{29}$ Thus, a key feature of FN assembly into fibers is its ability to undergo reversible conformational changes that convert soluble $\mathrm{FN}$ from its initial inactivated, compact conformation to a surface-activated, extended conformation. These features endow FN with its natural elasticity and extensibilities approaching $700 \%$ without failure and elastic moduli between 2-5 $\mathrm{MPa}^{30,31}$

Silk is another common protein that exhibits structure-dependent functional properties similar to collagen and FN but is not a protein found in mammals. ${ }^{16,24,32}$ Silk fibroin is native to spiders and silkworms, and contains alternating repetitive, hydrophobic core domains, rich in Alanine (A) and Glycine $(\mathrm{G})$, and a non-repetitive, hydrophilic C-terminal domain. ${ }^{8,15,33}$ During fibrillogenesis, fibroin monomers assemble into $\beta$-sheet crystallites that are stabilized by intramolecular hydrogen bonds. This core structure of crystallites contributes to silk's stiffness (5-7 GPa) and their extensibilities near $30 \% .{ }^{13,34}$ Like collagen, the high percentage of Glycine in silk enables a tightly packed secondary structure. However, the alternating repetitive and nonrepetitive primary structure of silk fibroin enables the protein to fold into a highly stable $\beta$-sheet secondary structure. ${ }^{16,24,32}$ Regardless of their final conformation, collagen, FN, and silk all possess repetitive sequences and domains stabilized by non-covalent bonds that contribute to the bulk mechanical properties of their formed fibers. These properties enable proteins to assemble into fibers endowed with a function dependent on ordered arrangements of their amino acids.

Why are these proteins noteworthy and the discussion of their properties timely? While we have manufactured with silk for decades, before protein fibers composed of proteins such as collagen or FN can be used in the textile industry, two technical challenges must be addressed: 1) 
protecting protein structure and function during the mass production of textiles, and 2) overcoming the limited natural abundance of the proteins themselves.

\section{Building Protein Textiles}

Commonly used fiber-forming manufacturing processes include melt, ${ }^{35,36} \mathrm{dry},{ }^{37,38}$ wet, ${ }^{39}$, 40 or electro ${ }^{41,42}$ spinning. These techniques involve solution extrusion under high pressures to form fibers and require a series of post-processing steps that include cooling gases (for melt and dry spinning) or precipitation (for wet spinning) to solidify formed fibers. ${ }^{9}$ Once the fibers are formed, they are collected, stretched, and aligned using spindles and reels. Electrospinning is a fabrication technique similar to conventional extrusion spinning, except that it utilizes an electric field $(5-20 \mathrm{kV})$ instead of tensile force to initiate polymer jet formation. ${ }^{9,41-43}$ Electrospun fibers form due to a potential difference between a charged starting solution and a grounded collector, where fibers solidify by evaporation. Despite its versatility to form polymer fibers, electrospinning is restricted by a low production rate $\left(0.5 \mathrm{~g} \mathrm{~h}^{-1}\right.$ per spinneret $)$ and poor control over fiber diameter and orientation. ${ }^{9}$ It is also dependent on solvent conductivity to direct the polymer jet to the collector substrate. $9,41,42,44,45$ These extensive post-processing techniques require that the starting material be robust enough to withstand these steps without denaturing, which is why most of these techniques are optimized to spin polymers, not proteins.

We argue that protein-based fibers will offer a wider range of functionalities, such as high extensibilities, biocompatibility, and tunable stiffness that are currently not available with polymer fibers. To spin proteins without denaturing their native structure or function, a processing technology must be developed to mimic the protein's natural state (i.e., aqueous environments and ambient temperatures). A biological model for protein fiber fabrication is 
spider silk fibrillogenesis (Figure 2A). This process is mediated by the interplay between ionic charges, $\mathrm{pH}$, and mechanical strain to produce silk microfibers with experimentally measured diameters of $19.7 \pm 2.8 \mu \mathrm{m}(\mathrm{N}=200$ fibers Figure 2A ii from Bombyx mori cocoons Figure 2A iii). During fibrillogenesis, soluble silk fibroin enters the spinning gland (ampulla) in an $\alpha$-helix conformation until it reaches the spinning duct (Figure 2B). Shear forces in the duct, along with a pH drop to 6.3 , alterations in ionic strength of the solution, and reduction of solution volumeall alter the hydration pattern of fibroin, which triggers its conformational change from $\alpha$-helix to $\beta$-sheet. ${ }^{12,} 13$ These $\beta$-sheet domains are stabilized by non-covalent interactions, which are assembled into fibers drawn from the spigot by the spider's hind legs, producing the silk web. ${ }^{8}$ 13, 46 Thus, the interplay between conformational changes, elongational flow, and shear stress yields the efficient production of mechanically stable, insoluble silk fibers.

We have developed two new forms of protein textile production that utilize either shear flow or conformational changes. ${ }^{44,} 47$ They offer appealing features, such as room-temperature processing, rapid prototyping, and high production rates without direct loss of protein function. The rotary jet spinning (RJS) technique was developed to manufacture polymer fibers using shear stress and elongational flow due to centrifugal forces in a mechanism that mimics how spiders extrude silk (Figure 2C i) ${ }^{44,48}$ The basic configuration of the RJS includes a reservoir containing a solution that is attached to a high-speed motor capable of rotational speeds between $0-75,000 \mathrm{RPM}$. As the reservoir rotates, the polymer solution is extruded through a $\sim 400 \mu \mathrm{m}$ orifice, and subsequent fibers are collected at a fixed distance away from the reservoir. ${ }^{44} \mathrm{We}$ hypothesize that these rotational speeds can be used to mimic the shear fluid flow in the spider duct to form insoluble silk fibers. To demonstrate the capabilities of the RJS to induce protein fibrillogenesis, silk fibroin protein was extracted from B. mori cocoons according to a previously 
published protocol $^{49}$ and solubilized in hexafluoro-2-propanol (3 wt\%). In its soluble form, silk fibroin has an $\alpha$-helical conformation. Once extruded under the shear forces of the RJS, fibroin assembles into insoluble nanofibers $(450 \pm 87 \mathrm{~nm}$ diameter, $\mathrm{N}=200$, Figure 2C ii). The secondary structure of native and RJS spun silk was measured using attenuated total reflectanceFourier transform infrared spectroscopy (ATR-FTIR, Figure 2C iii). Native B. mori silk fibers (blue) exhibit characteristic absorption peaks at 1516 and $1624 \mathrm{~cm}^{-1}$, representative of a $\beta$-sheet rich conformation. ${ }^{49}$ RJS produced nanofibers (black) similarly exhibit peaks at 1527 and 1635 $\mathrm{cm}^{-1}$, suggesting that these shear forces are large enough to induce fibrillogenesis. Collectively, these data indicate that shear fluid flow of RJS successfully mimics spider silk fibrillogenesis.

To better understand the mechanism of fibrillogenesis using the RJS, we developed an empirical model, where we identified three stages of fiber formation using the RJS: (1) jet initiation, which is dependent on reaching a defined threshold angular speed $(\Omega$, Figure $2 \mathbf{D}$ i), (2) jet extension, which is characterized as the balance of centrifugal and viscous forces shearing molecules within the jet (Figure 2D ii), and (3) solvent evaporation, which is dependent on diffusion of the solvent from the polymer (Figure 2D iii). ${ }^{44,48}$ Previous work has demonstrated that solution viscosity and rotational speed of the reservoir are the dominant parameters controlling fiber diameter. ${ }^{48}$ By tuning these parameters, highly aligned fibers with diameters ranging from 200 to $2000 \mathrm{~nm}$ can be formed under room temperature, aqueous conditions.

On a smaller size scale, protein nanoFabrics have been manufactured utilizing a surfaceinitiated assembly technique to induce conformational changes during fibrillogenesis (Figure 3). ${ }^{30,47}$ NanoFabrics are formed using micro-contact printing, a soft lithography technique used to deposit proteins in variety of defined patterns (Figure 3A). The process utilizes a high-density protein adsorption onto a hydrophobic stamp, followed by a transfer of the protein from the 
stamp to a temperature-sensitive substrate composed of a thin sacrificial polymer layer (Figure 3B). In the presence of a low temperature $\left(<32^{\circ} \mathrm{C}\right)$ aqueous solution, the sacrificial layer undergoes a phase transition from a solid to a liquid, releasing the fabrics from the substrate as free-standing fibrillar arrays (Figure 3C). This surface-initiated assembly triggers conformational changes in the proteins, which can then be used to expose unique mechanical properties. For instance, fabrics composed of $\mathrm{FN}$ have been shown to extend over $8 \mathrm{x}$ their original length without breaking via domain unfolding, illustrating that protein based textiles offer robust mechanical properties compared to current polymer textiles. This capability to engineer free-standing protein nanoFabrics with tunable composition, architecture, mechanical properties, and biological activity is an important proof-of-concept design tool for protein-based textiles.

The supply of natural proteins remains a major limitation in the manufacture of protein nanofibers. Currently, protein fibers are used "as harvested" directly from the source: wool from goats or sheep, silk from silkworm cocoons, and collagen from mouse or rat tails. In this state, proteins cannot be modified or altered, limiting their possible applications. To circumvent the limitations in harvested proteins, recombinant synthesis technologies have been optimized to not only enable protein production independent of the natural organism but also to provide the flexibility to design proteins de novo with strategically placed domains that will provide a specific function, such as elasticity, biocompatibility, or rigidity. ${ }^{17-19}$ Recombinant synthesis strategies utilize cells as machines to manufacture peptide sequences configured using computational modeling. ${ }^{18}$ The sequences can either directly mimic specific proteins or include selective domains within a protein. ${ }^{19}$ The configured sequence is used to construct a synthetic gene encoding polypeptide, which is inserted into a genetic vector and transferred to a host 
system. The host will then express the encoded polypeptide, and the polypeptide will be collected, purified, and characterized for further use.

Bacteria, such as Escherichia coli, are most common for recombinant expression of proteins, but these prokaryotic systems often fail to express specific folding patterns in complex, multi-modular proteins. Eukaryotic systems, such as yeast, have been used for transient expression of larger protein structures. ${ }^{17,19}$ Even so, yeast is only effective at expressing proteins that have fewer than 500 residues. Mammalian expression systems, such as Chinese hamster ovary (CHO) cells, human embryonic kidney (HEK) cells, or PER.C6 derived from human embryonic retina cells have been optimized to manufacture larger, extracellular matrix proteins, such as FN or collagen and have recently been optimized to yield milligram-scale quantities of protein from $\mathrm{CHO}$ and HEK cells and gram-scale quantities from PER.C6 cells. ${ }^{17,}$ 50-53 The advancements in recombinant protein technologies enable the production of engineered proteins that can be customized for a specific function. Higher yields of protein-based materials may also be realized by combining select protein domains with synthetic polymers through coupling reactions. ${ }^{54}$ These hybrid composites will maintain the functionality of proteins, while using only a fraction of the material.

\section{Testing the Mechanical Durability of Protein Textiles}

One of the novel features of protein fibers is their ability to bear tensile loads over extraordinary ranges without failing. Protein stability is defined as the unfolding force at a specific pulling velocity or loading rate, where larger forces denotes a more stable protein. ${ }^{55}$ Understanding how protein structure contributes to the bulk mechanical properties of a network requires tools sensitive enough to measure protein mechanics across multiple spatial scales 
(Figure 4). At the single protein level, experimental techniques, such as atomic force microscopy (AFM), have been used to measure the mechanical functions of proteins. AFM is a single molecule force technique that is capable of measuring $\mathrm{pN}$ forces generated by proteins or small molecules at a sub-nanometer spatial resolution utilizing a piezoelectric cantilever (Figure 4). ${ }^{55}$, 56 In AFM, the cantilever attaches to the protein of interest, which is adsorbed to a static substrate. As the protein is stretched, it becomes aligned with the applied force, and deflection at the protein-cantilever interface is used to calculate force generated during elongation. ${ }^{57-59}$ The force required to unfold proteins is dependent on the free-energy barrier $\left(\Delta \mathrm{G}_{\mathrm{T}-\mathrm{N}}\right)$. Once the initial barrier is crossed, a single domain begins to unfold, where the force threshold required to break all subsequent domains is smaller than the original. ${ }^{60,61}$ Proteins $\beta$-sheet secondary structures, such as FN or silk, display a sawtooth force-extension curve with mean force-peaks of 145-300 pN (Figure 4), where individual force peaks correspond to the mechanical unfolding of specific domains. ${ }^{56,58,60,62}$

Not only do protein unfolding forces depend the free-energy barrier $\left(\Delta \mathrm{G}_{\mathrm{T}-\mathrm{N}}\right)$, the intrinsic transition distance $(\Delta \mathrm{z})$ between folded and unfolded domains also regulates peak forces. The interplay between $\Delta \mathrm{G}_{\mathrm{T}-\mathrm{N}}$ and $\Delta \mathrm{z}$ in regulating unfolding forces can be understood using Bell's two state model. ${ }^{55,63}$ The two-state model considers a system being pulled over a free-energy barrier separated by two local minimal energy states and has previously been used to estimate protein unfolding under load. ${ }^{30,64}$ In this model:

$$
n_{f}=\frac{1}{1+\alpha_{0} \exp \left(F \Delta z / k_{B} T\right)}
$$

where

$$
n_{u}=1-n_{f}
$$


Here, $\alpha_{o}$ is the unfolding rate at zero force (or $\frac{n_{u}}{n_{f}}$ at zero force). The $n_{f}$ and $n_{u}$ variables are the fractions of folded and unfolded domains in a protein, respectively, $F$ is force, $k_{B} T$ is the thermal energy at room temperature $(T)$, and $\Delta z$ is the transition distance between the folded and unfolded states that may be collected experimentally through single-molecule force microscopy studies. Smaller unfolding rates $\left(\alpha_{o}\right)$ and shorter unfolding distances $(\Delta \mathrm{z})$ produce a larger $\Delta \mathrm{G}_{\mathrm{T}-\mathrm{N}}$ and higher unfolding force. For example, proteins enriched with $\beta$-sheet domains, such as FN or silk, are considered more stable than collagen because they require larger forces to overcome the $\Delta \mathrm{G}_{\mathrm{T}-\mathrm{N}}$ barrier due to the higher density of hydrogen bonds within their secondary structures. ${ }^{5,65}$ Thus, $\beta$-sheet domains stabilized by hydrogen bonding behave as a force barrier that resists protein unfolding. ${ }^{66}$ This differs from the force required to stretch $\alpha$-helix, which can unfold successively under force with no significant force peaks.

Computer simulations, such as steered molecular dynamics (SMD), are also used with AFM to understand how individual protein domains respond to mechanical strain. These simulations are atomistic reconstructions of protein primary structure designed to mimic experimentally measured single-molecule forces over a shorter time scale (SMD, $\sim \mu$ s and AFM, 1 s). ${ }^{65,66}$ SMD simulations suggest that larger, local forces (peak $\sim 1500 \mathrm{pN}$ ) are required to unfold $\beta$-sheets due to a higher density of intramolecular hydrogen bonds when compared to $\alpha$-helices (Figure 4). ${ }^{66}$ While SMD does provide useful quantitative measurements elucidating the relationship between mechanical strain and protein secondary structure, the shorter time scale of simulations often lead to larger force values compared to experimental values, suggesting that equilibrium is not reached during the time course of simulations. ${ }^{67}$ However, the combination of AFM and SMD together are useful in understanding the structure-dependent functional relationship of proteins. 
Proteins that assemble into fibers or networks of fibers often require $\mathrm{nN}-\mathrm{mN}$ forces to initiate elongation, and these properties can be measured using uniaxial tensile tests (Figure 4). ${ }^{30,68}$ For isolated fibers, these tests may be performed using finely tipped microneedles made of solid borosilicate glass rods or a piezoelectric MEMs force sensor. ${ }^{30,31,68}$ In these tests, the fiber is strained, and displacement at the microneedle-fiber or MEMs sensor-fiber interface is used to calculate force generated by the fiber under load. These techniques have been used to follow the strain dependent molecular changes in both FN and collagen protein networks. ${ }^{30,31,69}$ For a protein network, measurements can be recorded using an Instron mechanical tester. A standard Instron consists of two grips, which are used to suspend a fiber network in air or in solution (Figure 4). The fiber network is uniaxially loaded, which allows a direct measurement of elastic stress-strain behavior, young's modulus (E) and ultimate tensile strength (UTS). Depending on the protein secondary structure, fibers will exhibit a range of mechanical properties. Silk and collagen exhibit similar extensibilities $(\sim 30 \%)$, but silk is significantly stronger than collagen (silk $\sim 5 \mathrm{GPa}$, collagen $\sim 3 \mathrm{GPa}$ ), due to its $\beta$-sheet secondary structure and the larger $\Delta \mathrm{G}_{\mathrm{T}-\mathrm{N}}$ threshold to overcome during unfolding. ${ }^{13,26,34}$ On the other hand, FN is the softest of the three protein fibers $(\sim 5 \mathrm{MPa})$, even though it is embedded with $\beta$-sheets. ${ }^{30,} 31$ Because the FN III domains are not tightly packed like the $\beta$-sheet crystallites in silk, they can unfold/refold under load, contributing to FN's impressive $700 \%$ extensibility without breaking. From single domains to networks of fibers, the tools available to analyze the structure-dependent function of proteins contributes to defining parameters of molecules whose mechanical properties can be tuned depending on the arrangement and degree of structural domains that resist unfolding. 


\section{Summary and Outlook}

Our previous studies of protein self-assembly across multiple spatial scales have revealed two characteristics necessary for designing a mechanically stable protein mimetic: 1) repetitive primary structure and 2) a structure stabilized by non-covalent bonds. These components of a stable protein network must be engineered to realize the functionality of protein-based textiles for biological and non-biological applications. The span of spatial scales that require manufacturing control of these properties is formidable and will benefit from novel theoretical models of the materials, their scaling laws, manufacturing, and application. With these programmed characteristics, a new generation of smart textiles can be designed that will not only be soft and conformal but also mechanically stable. 
Acknowledgments: The authors gratefully acknowledge the use of facilities at the Harvard Center for Nanoscale Systems and the Wyss Institute for Biologically Inspired Engineering. We also acknowledge Professor Katia Bertoldi at Harvard University and Professor Jeffrey Ruberti at Northeastern University for their helpful discussions on protein mechanics and modeling. This work was partially funded by the Defense Advanced Research Projects Agency CINAPSE (W911NF-10-1-0113), and Harvard University Materials Research Science and Engineering Center supported by the National Science Foundation (DMR-0213805). 


\section{References}

1. L. Li, M. B. Charati and K. L. Kiick, Elastomeric polypeptide-based biomaterials. Polymer Chemistry 1, 1160 (2010).

2. K. Morris and L. Serpell, From natural to designer self-assembling biopolymers, the structural characterisation of fibrous proteins \& peptides using fibre diffraction. Chem. Soc. Rev. 39, 3445 (2010).

3. J. Sottile and D. C. Hocking, Fibronectin polymerization regulates the composition and stability of extracellular matrix fibrils and cell-matrix adhesions. Molecular Biology of the Cell 13, 3546 (2002).

4. J. E. Schwarzbauer and J. L. Sechler, Fibronectin fibrillogenesis: a paradigm for extracellular matrix assembly. Current Opinion in Cell Biology 11, 622 (1999).

5. V. Vogel, Mechanotransduction involving multimodular proteins: Converting force into biochemical signals. Annu. Rev. Biophys. Biomol. Struct. 35, 459 (2006).

6. F. Vollrath, D. Porter and C. Holland, There are many more lessons still to be learned from spider silks. Soft Matter 7, 9595 (2011).

7. J. W. S. Hearle, Protein fibers: structural mechanics and future opportunities. Journal of Materials Science 42, 8010 (2007).

8. M. Heim, D. Keerl and T. Scheibel, Spider Silk: From Soluble Protein to Extraordinary Fiber. Angewandte Chemie-International Edition 48, 3584 (2009).

9. C. J. Luo, S. D. Stoyanov, E. Stride, E. Pelan and M. Edirisinghe, Electrospinning versus fibre production methods: from specifics to technological convergence. Chem. Soc. Rev. 41, 4708 (2012).

10. M. L. Smith, D. Gourdon, W. C. Little, K. E. Kubow, R. A. Eguiluz, S. Luna-Morris and V. Vogel, Force-induced unfolding of fibronectin in the extracellular matrix of living cells. Plos Biology 5, 2243 (2007).

11. F. Vollrath and D. P. Knight, Structure and function of the silk production pathway in the Spider nephila edulis. Int. J. Biol. Macromol. 24, 243 (1999).

12. G. Askarieh, M. Hedhammar, K. Nordling, A. Saenz, C. Casals, A. Rising, J. Johansson and S. D. Knight, Self-assembly of spider silk proteins is controlled by a $\mathrm{pH}$-sensitive relay. Nature 465, 236 (2010).

13. D. Hochhalter, Artificially produced spider silk fibers as a high tech biological material. Basic Biotechnology 7, 12 (2011).

14. T. Gheysens, A. Collins, S. Raina, F. Vollrath and D. P. Knight, Demineralization Enables Reeling of Wild Silkmoth Cocoons. Biomacromolecules 12, 2257 (2011).

15. G. Gronau, S. T. Krishnaji, M. E. Kinahan, T. Giesa, J. Y. Wong, D. L. Kaplan and M. J. Buehler, A review of combined experimental and computational procedures for assessing biopolymer structure-process-property relationships. Biomaterials 33, 8240 (2012).

16. X. Hu, P. Cebe, A. S. Weiss, F. Omenetto and D. L. Kaplan, Protein-based composite materials. Mater. Today 15, 208 (2012).

17. A. R. Aricescu, W. Lu and E. Y. Jones, A time- and cost-efficient system for high-level protein production in mammalian cells. Acta Crystallographica Section D-Biological Crystallography 62, 1243 (2006).

18. E. D. Carlson, R. Gan, C. E. Hodgman and M. C. Jewett, Cell-free protein synthesis: Applications come of age. Biotechnol. Adv. 30, 1185 (2012).

19. R. L. DiMarco and S. C. Heilshorn, Multifunctional Materials through Modular Protein Engineering. Adv. Mater. 24, 3923 (2012).

20. B. Bondar, S. Fuchs, A. Motta, C. Migliaresi and C. J. Kirkpatrick, Functionality of endothelial cells on silk fibroin nets: Comparative study of micro- and nanometric fibre size. Biomaterials 29, 561 (2008). 
21. B. M. Min, L. Jeong, Y. S. Nam, J. M. Kim, J. Y. Kim and W. H. Park, Formation of silk fibroin matrices with different texture and its cellular response to normal human keratinocytes. Int. J. Biol. Macromol. 34, 281 (2004).

22. L. Soffer, X. Wang, X. Mang, J. Kluge, L. Dorfmann, D. L. Kaplan and G. Leisk, Silk-based electrospun tubular scaffolds for tissue-engineered vascular grafts. Journal of Biomaterials Science-Polymer Edition 19, 653 (2008).

23. C. Branden and J. Tooze, eds., Introduction to protein structure, 2nd edn., Garland Publishing, Inc, New York, 1991.

24. M. Heim, L. Romer and T. Scheibel, Hierarchical structures made of proteins. The complex architecture of spider webs and their constituent silk proteins. Chem. Soc. Rev. 39, 156 (2010).

25. K. E. Kadler, D. F. Holmes, J. A. Trotter and J. A. Chapman, Collagen fibril formation. Biochem. J 316, 1 (1996).

26. A. Gautieri, S. Vesentini, A. Redaelli and M. J. Buehler, Hierarchical Structure and Nanomechanics of Collagen Microfibrils from the Atomistic Scale Up. Nano Lett. 11, 757 (2011).

27. M. J. Buehler, Nanomechanics of collagen fibrils under varying cross-link densities: Atomistic and continuum studies. Journal of the Mechanical Behavior of Biomedical Materials 1, 59 (2008).

28. Y. Mao and J. E. Schwarzbauer, Fibronectin fibrillogenesis, a cell-mediated matrix assembly process. Matrix Biol. 24, 389 (2005).

29. I. Wierzbicka-Patynowski and J. E. Schwarzbauer, The ins and outs of fibronectin matrix assembly. J. Cell Sci. 116, 3269 (2003).

30. L. F. Deravi, T. Su, J. A. Paten, J. W. Ruberti, K. Bertoldi and K. K. Parker, Differential Contributions of Conformation Extension and Domain Unfolding to Properties of Fibronectin Nanotextiles. Nano Lett. 12, 5587 (2012).

31. E. Klotzsch, M. L. Smith, K. E. Kubow, S. Muntwyler, W. C. Little, F. Beyeler, D. Gourdon, B. J. Nelson and V. Vogel, Fibronectin forms the most extensible biological fibers displaying switchable force-exposed cryptic binding sites. Proceedings of the National Academy of Sciences of the United States of America 106, 18267 (2009).

32. G. H. Altman, F. Diaz, C. Jakuba, T. Calabro, R. L. Horan, J. S. Chen, H. Lu, J. Richmond and D. L. Kaplan, Silk-based biomaterials. Biomaterials 24, 401 (2003).

33. S. Keten, Z. P. Xu, B. Ihle and M. J. Buehler, Nanoconfinement controls stiffness, strength and mechanical toughness of beta-sheet crystals in silk. Nature Materials 9, 359 (2010).

34. M. A. Serban, J. A. Kluge, M. M. Laha and D. L. Kaplan, Modular Elastic Patches: Mechanical and Biological Effects. Biomacromolecules 11, 2230 (2010).

35. K. Mezghani and J. E. Spruiell, High speed melt spinning of poly(L-lactic acid) filaments. Journal of Polymer Science Part B-Polymer Physics 36, 1005 (1998).

36. K. P. Matabola, A. R. De Vries, F. S. Moolman and A. S. Luyt, Single polymer composites: a review. Journal of Materials Science 44, 6213 (2009).

37. S. M. Berry, S. A. Harfenist, R. W. Cohn and R. S. Keynton, Characterization of micromanipulator-controlled dry spinning of micro- and sub-microscale polymer fibers. Journal of Micromechanics and Microengineering 16, 1825 (2006).

38. W. Wei, Y. P. Zhang, Y. M. Zhao, J. Luo, H. L. Shao and X. C. Hu, Bio-inspired capillary dry spinning of regenerated silk fibroin aqueous solution. Materials Science \& Engineering CMaterials for Biological Applications 31, 1602 (2011).

39. D. M. Phillips, L. F. Drummy, R. R. Naik, H. C. De Long, D. M. Fox, P. C. Trulove and R. A. Mantz, Regenerated silk fiber wet spinning from an ionic liquid solution. J. Mater. Chem. 15, 4206 (2005).

40. K. D. Nelson, A. Romero, P. Waggoner, B. Crow, A. Borneman and G. M. Smith, Technique paper for wet-spinning Poly(L-lactic acid) and poly(DL-lactide-co-glycolide) monofilament fibers. Tissue Eng. 9, 1323 (2003). 
41. Z. M. Huang, Y. Z. Zhang, M. Kotaki and S. Ramakrishna, A review on polymer nanofibers by electrospinning and their applications in nanocomposites. Compos. Sci. Technol. 63, 2223 (2003).

42. Q. P. Pham, U. Sharma and A. G. Mikos, Electrospinning of polymeric nanofibers for tissue engineering applications: A review. Tissue Eng. 12, 1197 (2006).

43. D. H. Reneker and A. L. Yarin, Electrospinning jets and polymer nanofibers. Polymer 49, 2387 (2008).

44. M. R. Badrossamay, H. A. McIlwee, J. A. Goss and K. K. Parker, Nanofiber Assembly by Rotary Jet-Spinning. Nano Lett. 10, 2257 (2010).

45. J. E. Plowman, S. Deb-Choudhury, S. Clerens, A. Thomas, C. D. Cornellison and J. M. Dyer, Unravelling the proteome of wool: Towards markers of wool quality traits. Journal of Proteomics 75, 4315 (2012).

46. F. G. Omenetto and D. L. Kaplan, New Opportunities for an Ancient Material. Science 329, 528 (2010).

47. A. W. Feinberg and K. K. Parker, Surface-Initiated Assembly of Protein Nanofabrics. Nano Lett. 10, 2184 (2010).

48. P. Mellado, H. A. McIlwee, M. R. Badrossamay, J. A. Goss, L. Mahadevan and K. K. Parker, A simple model for nanofiber formation by rotary jet-spinning. Appl. Phys. Lett. 99 (2011).

49. C. M. Li, C. Vepari, H. J. Jin, H. J. Kim and D. L. Kaplan, Electrospun silk-BMP-2 scaffolds for bone tissue engineering. Biomaterials 27, 3115 (2006).

50. M. I. Cockett, C. R. Bebbington and G. T. Yarranton, High-level Expression of Tissue Inhibitor of Metalloproeinases in Chinese Hamster Ovary Cells using Glutamine-synthetase Gene Amplification. Bio-Technology 8, 662 (1990).

51. F. Mancia, S. D. Patel, M. W. Rajala, P. E. Scherer, A. Nemes, I. Schieren, W. A. Hendrickson and L. Shapiro, Optimization of protein production in mammalian cells with a coexpressed fluorescent marker. Structure 12, 1355 (2004).

52. S. Geisse and M. Henke, Large-scale transient transfection of mammalian cells: A newly emerging attractive option for recombinant protein production. Journal of Structural and Functional Genomics 6, 165 (2005).

53. M. Butler and A. Meneses-Acosta, Recent advances in technology supporting biopharmaceutical production from mammalian cells. Appl. Microbiol. Biotechnol. 96, 885 (2012).

54. B. Jung and P. Theato, Chemical Strategies for the Synthesis of Protein-Polymer Conjugates. Adv. Polym. Sci. 253, 37 (2013).

55. H. B. Li, 'Mechanical Engineering' of Elastomeric Proteins: Toward Designing New Protein Building Blocks for Biomaterials. Adv. Funct. Mater. 18, 2643 (2008).

56. E. M. Puchner and H. E. Gaub, Force and function: probing proteins with AFM-based force spectroscopy. Current Opinion in Structural Biology 19, 605 (2009).

57. P. E. Marszalek, H. Lu, H. B. Li, M. Carrion-Vazquez, A. F. Oberhauser, K. Schulten and J. M. Fernandez, Mechanical unfolding intermediates in titin modules. Nature 402, 100 (1999).

58. A. F. Oberhauser, C. Badilla-Fernandez, M. Carrion-Vazquez and J. M. Fernandez, The mechanical hierarchies of fibronectin observed with single-molecule AFM. J. Mol. Biol. 319, 433 (2002).

59. M. Carrion-Vazquez, A. F. Oberhauser, S. B. Fowler, P. E. Marszalek, S. E. Broedel, J. Clarke and J. M. Fernandez, Mechanical and chemical unfolding of a single protein: A comparison. Proceedings of the National Academy of Sciences of the United States of America 96, 3694 (1999).

60. M. Rief, M. Gautel, A. Schemmel and H. E. Gaub, The mechanical stability of immunoglobulin and fibronectin III domains in the muscle protein titin measured by atomic force microscopy. Biophys. J. 75, 3008 (1998).

61. M. Rief, J. Pascual, M. Saraste and H. E. Gaub, Single molecule force spectroscopy of spectrin repeats: Low unfolding forces in helix bundles. J. Mol. Biol. 286, 553 (1999). 
62. D. B. Staple, S. H. Payne, A. L. C. Reddin and H. J. Kreuzer, Model for Stretching and Unfolding the Giant Multidomain Muscle Protein Using Single-Molecule Force Spectroscopy. Phys. Rev. Lett. 101 (2008).

63. G. I. Bell, Models for specific adhesion of cells to cells. Science 200, 618 (1978).

64. A. E. X. Brown, R. I. Litvinov, D. E. Discher, P. K. Purohit and J. W. Weisel, Multiscale Mechanics of Fibrin Polymer: Gel Stretching with Protein Unfolding and Loss of Water. Science 325, 741 (2009).

65. E. P. S. Gee, D. E. Ingber and C. M. Stultz, Fibronectin Unfolding Revisited: Modeling Cell Traction-Mediated Unfolding of the Tenth Type-III Repeat. Plos One 3 (2008).

66. H. Lu and K. Schulten, Steered molecular dynamics simulations of force-induced protein domain unfolding. Proteins-Structure Function and Genetics 35, 453 (1999).

67. Z. Lu, H. Hu, W. Yang and P. E. Marszaleky, Simulating force-induced conformational transitions in polysaccharides with the SMD replica exchange method. Biophys. J. 91, L57 (2006).

68. B. P. Flynn, A. P. Bhole, N. Saeidi, M. Liles, C. A. DiMarzio and J. W. Ruberti, Mechanical strain stabilizes reconstituted collagen fibrils against enzymatic degradation by mammalian collagenase matrix metalloproteinase 8 (MMP-8). Plos One 5 (2010).

69. J. A. Paten, G. E. Tilburey, E. A. Molloy, R. Zareian, C. V. Trainor and J. W. Ruberti, Utility of an optically-based, micromechanical system for printing collagen fibers. Biomaterials 34, 2577 (2013). 


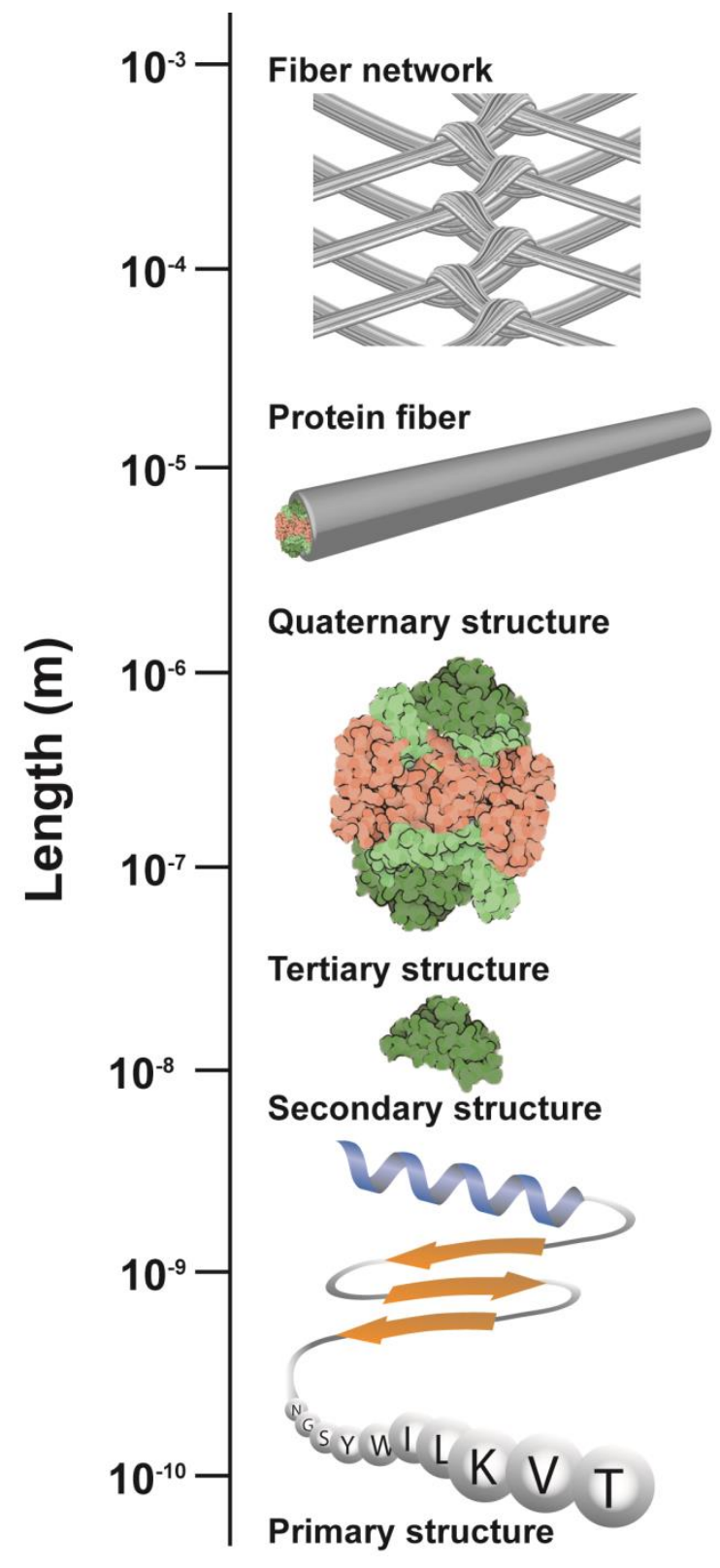

Figure 1. Spatial scaling of protein networks. The structure-dependent functional properties of protein networks begin with the assembly of amino acids that form the primary structure that regulate the coordinated assembly of protein fibers to form networks. 

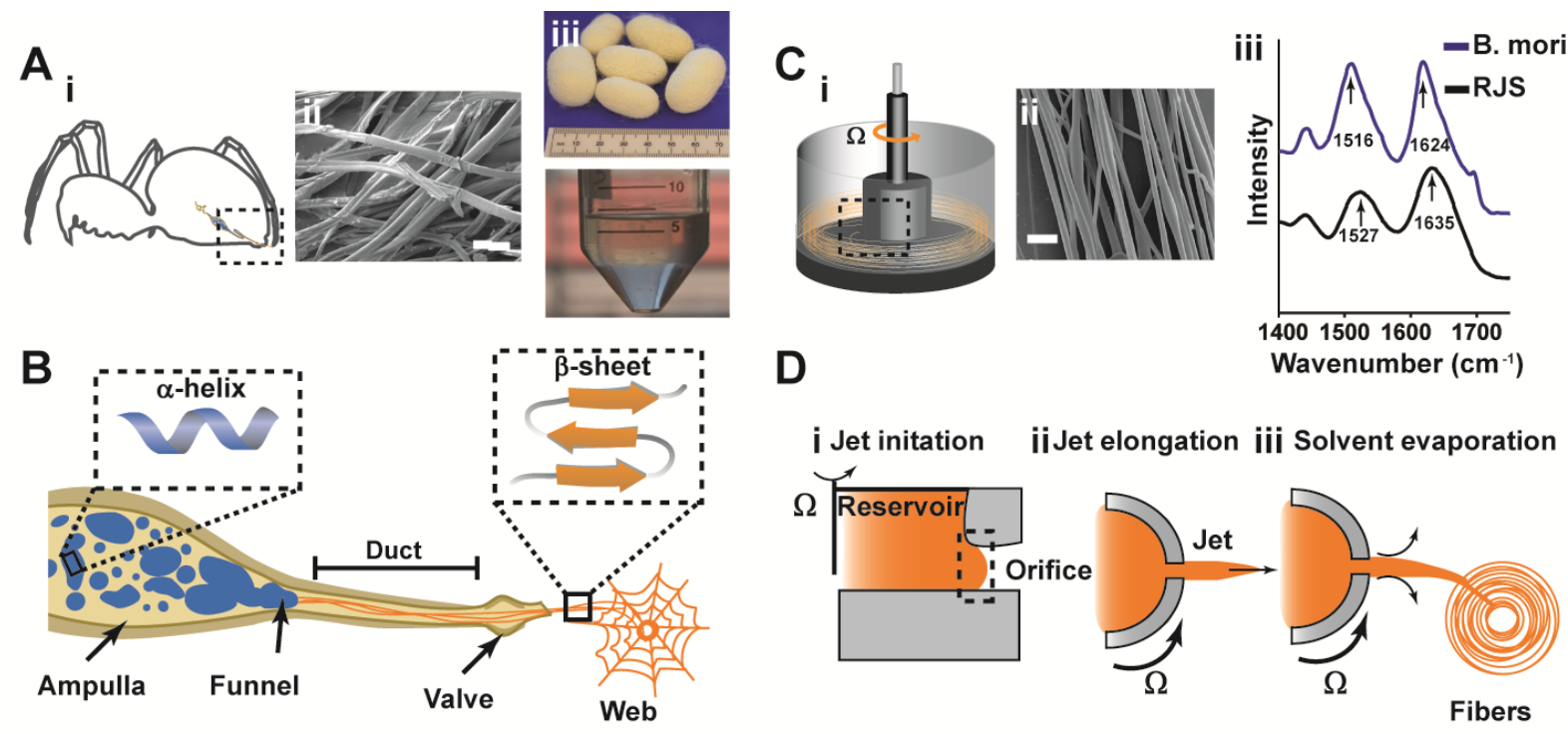

D

i Jet initation iiJet elongation iii Solvent evaporation
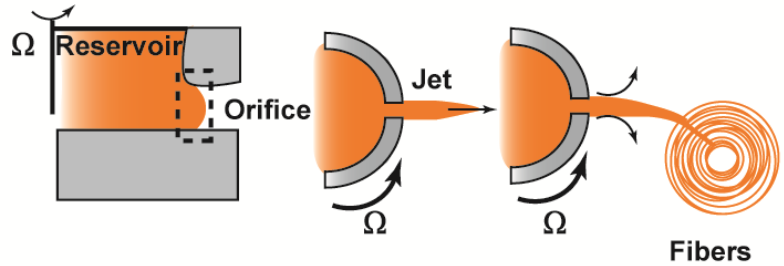

Figure 2. Spider silk fibrillogenesis as a model for fiber fabrication. A) Spiders synthesize silk fibers through solution extrusion, providing a biological model for fibrillogenesis (i). (ii) Scanning electron micrograph (SEM) of as formed silk fibroin fibers (scale bar, $40 \mu \mathrm{m}$ ) from Bombyx mori silkworm cocoons (iii). (iv) Soluble silk fibroin proteins extracted from cocoons. B) Schematic of the functional anatomy of the silk gland and spinning duct. The ampulla is the storage sac of the silk gland. The funnel orients the protein while it enters the duct. The proximal and medial limbs of the duct function to remove water, reducing total volume during fibrillogenesis. The valve is the pump that begins the spinning process. C) Rotary jet spinning (RJS) as a bio-inspired model of spider silk fibrillogenesis. (i) A schematic of the RJS device, where $\Omega$ is the angular speed. (ii) SEM of silk fibers as produced by the RJS (scale bar, $2 \mu \mathrm{m}$ ). Silk fibroin is dissolved in $3 \mathrm{wt} \%$ hexafluoro-2-propanol, and fibers are spun at 75,000 rpm. (iii) ATR-FTIR spectra of B. mori silk (blue) and RJS silk spun at 20,000 RPM (black). D)

Schematic of fiber formed using the RJS reservoir. (i) Side view of jet initiation. (ii-iii) top view of jet elongation and solvent evaporation to form fibers. 

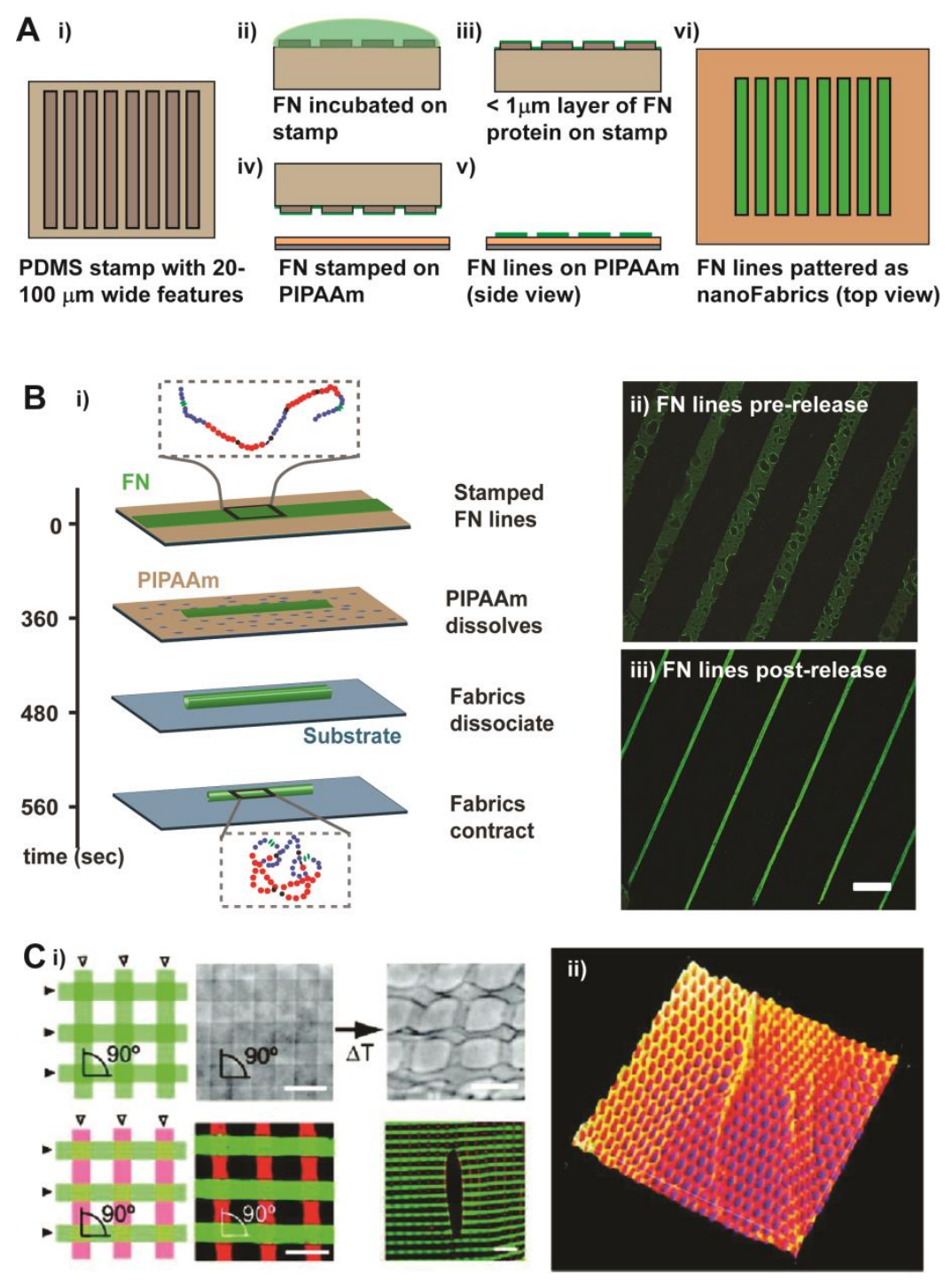

Figure 3. Surface initiated assembly of FN nanoFabrics. A) FN fibers are patterned using micro-contact printing. (i) This process utilizes a PDMS stamp engraved with micro-scale features inked with a protein (FN) of interest (ii). (iii) The features of the stamp are coated with a thin layer of protein and transferred to a thermosensitive PIPAAm coated glass substrate (iv). (vvi) The resultant pattern is used to form nanoFabrics. B) FN nanoFabrics are fibrillar arrays formed after they are released from the PIPAAm substrate. (i) This process takes advantage of conformational changes in FN. In the presence of low temperature aqueous solutions, PIPAAm changes its phase, to release the micro-patterned FN (ii) as fibers (iii). Scale bar is $100 \mu \mathrm{m}$. Reprinted with permission from Nano Lett. 2012, 12, 5587-5592. Copyright 2012 American Chemical Society. C) This process is amenable to a number of different patterns and proteins (i). Red is laminin lines and green is fibrinogen. ii) SEM image representing a three-dimensional FN nanoFabric whose $\mathrm{X}$ and $\mathrm{Y}$ axes are $360 \mu \mathrm{m}$. Reprinted with permission from Nano Lett. 2010, 10, 2184--2191. Copyright 2010 American Chemical Society. 


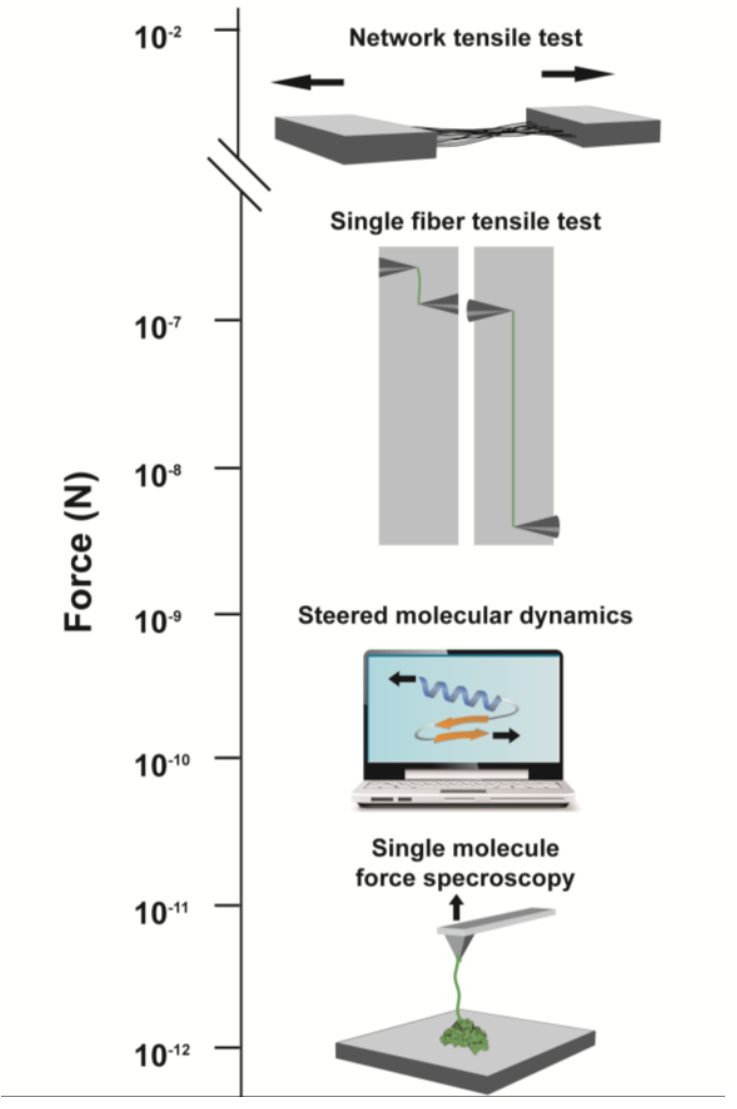

Figure 4. Spatial scaling of mechanical tools available to study protein mechanics. The mechanical properties of proteins can be characterized across multiple spatial scales beginning with experimental measured force-extension curves using AFM coupled with SMD simulations at $\mathrm{pN}$ forces to the bulk mechanical properties of a protein network using uniaxial tensile testing at $\mathrm{mN}$ forces. 